\title{
Introito calorico e nutrizionale in un gruppo di pazienti con trapianto di rene
}

Giornale di Tecniche Nefrologiche e Dialitiche 2018, Vol. 30(2) 105-110

(C) The Author(s) 2018

Article reuse guidelines:

sagepub.com/journals-permissions DOI: $10.1177 / 0394936218807900$ journals.sagepub.com/home/gtn

\section{(S)AGE}

\section{Patrizia Calella', Claudia D’Alessandro², Domenico Giannese'2, Massimiliano Barsotti ${ }^{2}$ and Vincenzo Bellizzi ${ }^{3}$}

\begin{abstract}
Nutritional intakes in Italian kidney transplant recipients
Background and Aim: Obesity, dyslipidemia, hypertension and glucose intolerance are some of the main nutritional and metabolic problems that occur in post-transplant patients. We evaluated the daily dietary intake in renal transplant recipients to assess whether they fulfil the nutrient intake recommendations (according to the Italian recommended dietary allowances, LARN 2014).

Subjects and Methods: Renal allograft recipients that were transplanted between 6 months and 5 years before this study were recruited. The daily intake of nutrients, including protein and salt, was estimated with a five-day food diary. Results: In total, 50 patients were studied ( 31 males and I 9 females); the mean patients' age was $48.9 \pm 9.9$ years, the mean age at transplant was $20.5 \pm 15.2$ years (median $15.3 \mathrm{y}$ ) and the mean eGFR was $56.4 \pm 20.2 \mathrm{~mL} / \mathrm{min} / 1.73 \mathrm{~m}^{2}$, with the eGFR terziles defined as $>66.7$, between 44.6 and 66.7 , and $<44.6 \mathrm{~mL} / \mathrm{min} / 1.73 \mathrm{~m}^{2}$. The mean body mass index was $26.7 \pm 4.4 \mathrm{~kg} / \mathrm{m}^{2}$. The medium total energy intake was $27.6 \pm 7.6 \mathrm{kcal} / \mathrm{kg}$ body weight $/$ day. The daily intake of proteins and salt were respectively $1.06 \pm 0.32 \mathrm{~g} / \mathrm{kg}$ body weight/day and $1.203 \mathrm{mg} /$ day. The calcium, iron and fiber intakes were respectively $484 \pm 236 \mathrm{mg} / \mathrm{die}, 9.6 \pm 2.9 \mathrm{mg} / \mathrm{die}$, and $12.2 \pm 4.9 \mathrm{~g} / \mathrm{die}$. No differences in nutrients intake were observed between patients with different degree of renal insufficiency; also, protein intake was above the threshold of $1.0 \mathrm{~g} / \mathrm{kgBW} /$ day in all patients.

Discussion: Our findings suggest that renal transplant recipients in Italy seem to have an adequate energy intake, with normal distributions of proteins, lipids and carbohydrates; the protein intake, however, was higher than that recommended for chronic kidney disease (CKD), at least for moderate to advanced CKD. On the contrary, the daily intake of fiber, calcium and iron were insufficient. Finally, the salt intake was greater than the recommendation in almost all individuals.

Conclusions: In renal transplant patients the mean dietary intakes fit with the recommendations, though this is not true for all the patients studied especially regarding the salt and protein intakes in the moderate to advance CKD stages. This observation suggests the need of a specific nutritional counselling and a regular nutritional assessment in patients with renal transplantation.
\end{abstract}

\section{Keywords}

Kidney transplantation, Renal transplantation, Diet, Dietary habits, Nutrients intake, CKD

\footnotetext{
'Department of Movement Sciences and Wellbeing, Parthenope University, Naples, Italy

${ }^{2}$ Department of Clinical and Experimental Medicine, University of Pisa, Pisa, Italy

${ }^{3}$ Division of Nephrology, Dialysis and Renal Transplantation, Nephrology Unit, University Hospital "San Giovanni di Dio e Ruggi d'Aragona", Salerno, Italy
}

\author{
Corrispondenza: \\ Dr. Vincenzo Bellizzi, MD, PhD, Division of Nephrology, Dialysis and \\ Renal Transplantation, University Hospital "San Giovanni di Dio e Ruggi \\ d'Aragona", Via San Leonardo, 84I3I Salerno, Italy. \\ Email: vincenzo.bellizzi@tin.it
}




\section{Introduzione}

I soggetti portatori di trapianto renale (TxR) hanno un aumentato rischio di patologie cardiovascolari rispetto alla popolazione generale di pari età. Questo è dovuto sia all'alta comorbidità cardiovascolare pre-trapianto tipica della malattia renale cronica (MRC) che agli effetti negativi della terapia immunosoppressiva. ${ }^{1-3}$ Per ridurre il rischio cardiovascolare è necessario ottimizzare la terapia immunosoppressiva ed instaurare uno stile di vita sano, preservando una composizione corporea normale e un buono stato nutrizionale.

Un'alimentazione corretta e bilanciata ha un ruolo centrale nella prevenzione delle malattie cardiovascolari, anche in soggetti affetti da insufficienza renale cronica, inclusi i pazienti con TxR. ${ }^{4-6}$ Dopo TxR, le migliori condizioni generali del paziente, il recupero della funzione renale e l'utilizzo di steroidi aumentano l'appetito e le quantità di cibo ingerito, inducendo spesso un rapido aumento del peso corporeo. Tuttavia, l'insorgenza o il peggioramento di complicanze cardiovascolari nel postTxR correla con l'indice di massa corporea (IMC); per questo motivo prevenire l'aumento di peso controllando l'apporto energetico è un intervento essenziale nella prevenzione delle complicanze cardiovascolari. ${ }^{7}$ L'introito in eccesso di specifici nutrienti, come ad esempio i grassi e il sale, che svolgono un ruolo principale rispettivamente nello sviluppo della dislipidemia e dell'ipertensione arteriosa, contribuisce allo sviluppo dei principali fattori di rischio cardiovascolare nel trapianto renale.

I problemi nutrizionali dei pazienti con TxR possono essere anche rappresentati da un difetto alimentare, piuttosto che dall'eccesso di nutrienti. Prima del trapianto, ad esempio durante le fasi di terapia dialitica sostitutiva o conservativa della MRC, vengono prescritte al paziente severe restrizioni alimentari; se queste abitudini alimentari dovessero continuare dopo il trapianto, anche considerando la concomitante condizione di iper-catabolismo, questo aumenterebbe il rischio di malnutrizione. ${ }^{8,9}$

L'obiettivo di un intervento nutrizionale nei pazienti trapiantati, dunque, è duplice: (I) trattare la malnutrizione (proteico-energetica) preesistente, evitando così una nutrizione in difetto da una parte e prevenendo l'eccesivo aumento ponderale dall'altra; (II) controllare l'introito di quantità inadeguate di macro e micronutrienti che possono avere un impatto negativo sul rischio cardiovascolare del paziente. Le linee guida nutrizionali in $\mathrm{TxR}^{10-12}$ suggeriscono un approccio nutrizionale ipercalorico e iperproteico nell'immediato post-trapianto per poi tendere progressivamente a normalizzare gli introiti verso i livelli della popolazione generale dopo alcune settimane o mesi dall'intervento. In fase di trapianto stabile e con buona funzionalità d'organo le restrizioni nutrizionali per fosforo, potassio e quantità di liquidi sono limitate ai casi in cui ci siano specifiche alterazioni cliniche che rendano necessari interventi restrittivi. Quando poi dovesse esserci una riduzione della funzione renale, allora le proteine alimentari dovrebbero essere controllate, almeno evitando introiti superiori ai valori consigliati per la popolazione generale. ${ }^{13-15}$

Sebbene molti studi abbiano indicato l'importanza di una corretta gestione nutrizionale nei pazienti con TxR, dati relativi alle abitudini alimentari di tali pazienti in Italia sono piuttosto limitati. Lo scopo di questo studio è quello di esaminare gli introiti nutrizionali in una popolazione di TxR adulti.

\section{Metodi}

Sono stati studiati pazienti con TxR ricevuto da 6 mesi a 5 anni prima dello studio e che presentavano condizioni cliniche stabili e in regolare follow-up ambulatoriale. Le abitudini alimentari e l'introito calorico, nonché di macro e micronutrienti, sono stati valutati usando un diario alimentare per 5 giorni (tre giorni feriali più sabato $\mathrm{e}$ domenica). Il diario alimentare è stato consegnato ai pazienti da una dietista che ha spiegato loro le modalità di compilazione, indicando l'importanza di pesare a crudo gli alimenti consumati abitualmente, di non cambiare le proprie abitudini alimentari né le porzioni delle pietanze. Ė stato chiesto di indicare le marche dei prodotti commerciali utilizzati per favorire un calcolo più accurato dei nutrienti. Alla riconsegna del diario esso veniva controllato e la dietista eseguiva il recall approfondendo i dati mancanti o poco chiari. Il calcolo degli apporti di calorie e di nutrienti è stato effettuato utilizzando il software Metadieta 3.8; i valori ottenuti sono stati confrontati con i valori di riferimento proposti per la popolazione generale in base ai Livelli di Assunzione di Riferimento di Nutrienti ed energia della popolazione generale italiana (LARN). ${ }^{16}$

Il protocollo di studio è stato approvato dal Comitato Etico dell'Azienda Ospedaliera Universitaria "San Giovanni di Dio e Ruggi d'Aragona" di Salerno, e i pazienti arruolati hanno rilasciato consenso informato per la partecipazione allo studio.

I dati sono stati analizzati con il software statistico Spss 24.0 e i dati sono riportati come media \pm 1 deviazione standard; il confronto tra i gruppi è stato effettuato con il test di Student e le differenze sono state considerate significative per valori di $\mathrm{p}<0.05$.

\section{Risultati}

Sono stati studiati 50 pazienti, 31 maschi e 19 femmine, con età media di 49 anni (range 27-63 anni); peso e altezza erano maggiori nei maschi ma questa differenza si annullava quando si valutava l'indice di massa corporea (IMC); l'età media del trapianto era simile nei due generi; il filtrato stimato risultava essere leggermente maggiore nei maschi (tabella 1). In base alle categorie di IMC 
Tabella I. Caratteristiche generali dei pazienti portatori di trapianto renale; popolazione totale e divisa per genere.

\begin{tabular}{|c|c|c|c|c|c|}
\hline & & Totale & Maschi & Femmine & $P$ \\
\hline Campione & $n$ & 50 & 31 & 19 & \\
\hline Età & anni & $48.9 \pm 9.9$ & $48.9 \pm 10.8$ & $48.9 \pm 8.7$ & 0.969 \\
\hline Altezza & $m t$ & $1.7 \pm 0.1$ & $1.7 \pm 0.1$ & $1.6 \pm 0.1$ & 0.000 \\
\hline Peso & $\mathrm{kg}$ & $73.5 \pm 14.0$ & $77.7 \pm 12.1$ & $66.6 \pm 14.6$ & 0.050 \\
\hline IMC & $\mathrm{kg} / \mathrm{m}^{2}$ & $26.7 \pm 4.4$ & $27.1 \pm 4.1$ & $26.2 \pm 4.9$ & 0.492 \\
\hline Età TxR & mesi & $20.5 \pm 15.2$ & $20.3 \pm 15.7$ & $20.8 \pm 14.8$ & 0.904 \\
\hline Creatinina & $\mathrm{mg} / \mathrm{dL}$ & $1.45 \pm 0.49$ & $1.46 \pm 0.45$ & $1.42 \pm 0.6$ & 0.788 \\
\hline eGFR-MDRD & $\mathrm{mL} / \mathrm{min} / \mathrm{l} .73 \mathrm{~m}^{2}$ & $56.4 \pm 20.2$ & $60.8 \pm 19.6$ & $49.2 \pm 19.4$ & 0.046 \\
\hline
\end{tabular}

definite dall'Organizzazione Mondiale della Sanità ${ }^{17}$, il $2 \%$ del campione era sottopeso (IMC $<18.5$ ), il $34 \%$ normopeso (IMC compreso tra18.5 e 24.9), il $40 \%$ sovrappeso (IMC tra 25 e 29.9 ) e il rimanente $24 \%$ era obeso (IMC $>30$ ) (figura 1). L'IMC non correlava con la durata del trapianto $(\mathrm{R}=-0.022 ; \mathrm{p}=0.881)$.

L'introito medio di calorie e principali macro e micronutrienti è mostrato in tabella 2, per l'intero campione di studio e per sottogruppi di genere e di funzionalità renale in base ai terzili di eGFR. L'introito medio di calorie e proteine per peso corporeo era rispettivamente di 27.6 \pm 7.6 $\mathrm{kcal} / \mathrm{kg}$ e $1.06 \pm 0.32 \mathrm{~g} / \mathrm{kg}$ al giorno; l'introito energetico era maggiore nei maschi $(\mathrm{p}<0.001)$. Nei maschi si osservava anche un apporto più elevato di grassi, e tra questi un più alto apporto di monoinsaturi e polinsaturi, nonché di carboidrati totali $(\mathrm{p}<0.05)$. Tra i micronutrienti, solo per il ferro si osservava una significativa differenza tra maschi e femmine $(p<0.01)$ con un introito ridotto nelle femmine. Dividendo i pazienti in base al terzile di funzione renale, rispettivamente eGFR $<44.6$, eGFR compreso tra 44.6 e 66.7, e eGFR $>66.7 \mathrm{~mL} / \mathrm{min} / 1.73 \mathrm{~m}^{2}$, non si osservava alcuna differenza significativa di introiti nutrizionali, eccetto per il colesterolo totale che era maggiore nel secondo terzile rispetto agli altri due $(\mathrm{p}=0.04)$. L'introito proteico era maggiore di $1 \mathrm{gr} / \mathrm{kg}$ di peso al giorno in tutti i terzili di eGFR; nel terzile alto questo introito corrisponde alle indicazioni per la MRC, mentre negli altri due terzili è superiore alle indicazioni.

In Figura 2 viene mostrata la distribuzione percentuale dell'intera popolazione di studio in relazione ai valori raccomandati per i principali macro e micronutrienti. In dettaglio, il $42 \%$ dei soggetti assumeva un quantitativo maggiore di calorie rispetto al range di riferimento consigliato, il $58 \%$ assumeva più proteine, il $34 \%$ più lipidi e il $46 \%$ più sodio; quasi tutti i pazienti non soddisfacevano i fabbisogni di fibre e calcio, mentreil $54 \%$ non soddifaceva i fabbisogni di ferro. L'introito di ferro non correlava con $\mathrm{i}$ valori di emoglobina $(\mathrm{R}=0.176$; $\mathrm{p}=0.221$ ).

In tabella 3 sono riportati il numero totale e la classe di farmaci immunosoppressori assunti dall'intero campione e dai sottogruppi suddivisi per stadio di malattia renale. In particolare, la maggior parte dei pazienti assumeva tre farmaci immunosoppressori: tutti i pazienti erano in trattamento con steroidi, quasi tutti i pazienti assumevano un inibitore delle calcineurine (con una tendenza alla prevalenza del Tacrolimus nei pazienti con funzione renale più conservata e di Ciclosporina nelle fasi più avanzate di malattia), e circa l' $80 \%$ dei pazienti assumeva Micofenolato senza sostanziale differenza tra i gruppi di funzione renale.

\section{Discussione}

Questo studio valuta l'introito di energia e dei principali nutrienti in un gruppo di individui portatori di TxR. In questi pazienti gli introiti medi di energia e dei principali nutrienti sono risultati nel range considerato di normalità per la popolazione generale, seppure con una variabilità piuttosto ampia, tanto che dal $30 \%$ al $50 \%$ dei pazienti superava gli introiti consigliati. Inoltre, abbiamo osservato che l'introito di proteine e di alcuni micronutrienti riscontrati in questi pazienti non corrisponde alle indicazioni per la malattia renale cronica; infatti, l'introito proteico era sempre superiore al grammo per kilo di peso al giorno per ogni grado di funzione renale e, dunque, adeguato per i pazienti con funzione renale normale ma elevato per i pazienti con MRC avanzata.

L'intervallo di IMC del campione di TxR studiato era compreso tra 17 e 35 , indicando quindi una grande variabilità. Solo il $34 \%$ dei pazienti era normopeso mentre il $64 \%$ era sovrappeso oppure obeso. L'obesità e il sovrappeso sono problemi comuni nei pazienti con TxR e si associano ad un aumentato rischio di malattie cardiovascolari, ipertensione, dislipidemia e diabete. ${ }^{18}$ Precedenti osservazioni hanno descritto un'incidenza tra il $15 \%$ e il $23 \%$ di soggetti con segni di iponutrizione, indicata da un IMC $<21 \mathrm{~kg} / \mathrm{m}^{2}$, con aumentato rischio di perdita della funzione renale. ${ }^{19,20}$ In questo campione di studio, la percentuale di soggetti con un IMC $<18.5 \mathrm{~kg} /$ $\mathrm{m}^{2}$, indicativo di malnutrizione, era del $2 \%$, quindi relativamente bassa rispetto ad altre casistiche. Tuttavia, circa 2 pazienti su 3 erano sovrappeso oppure obesi, e 


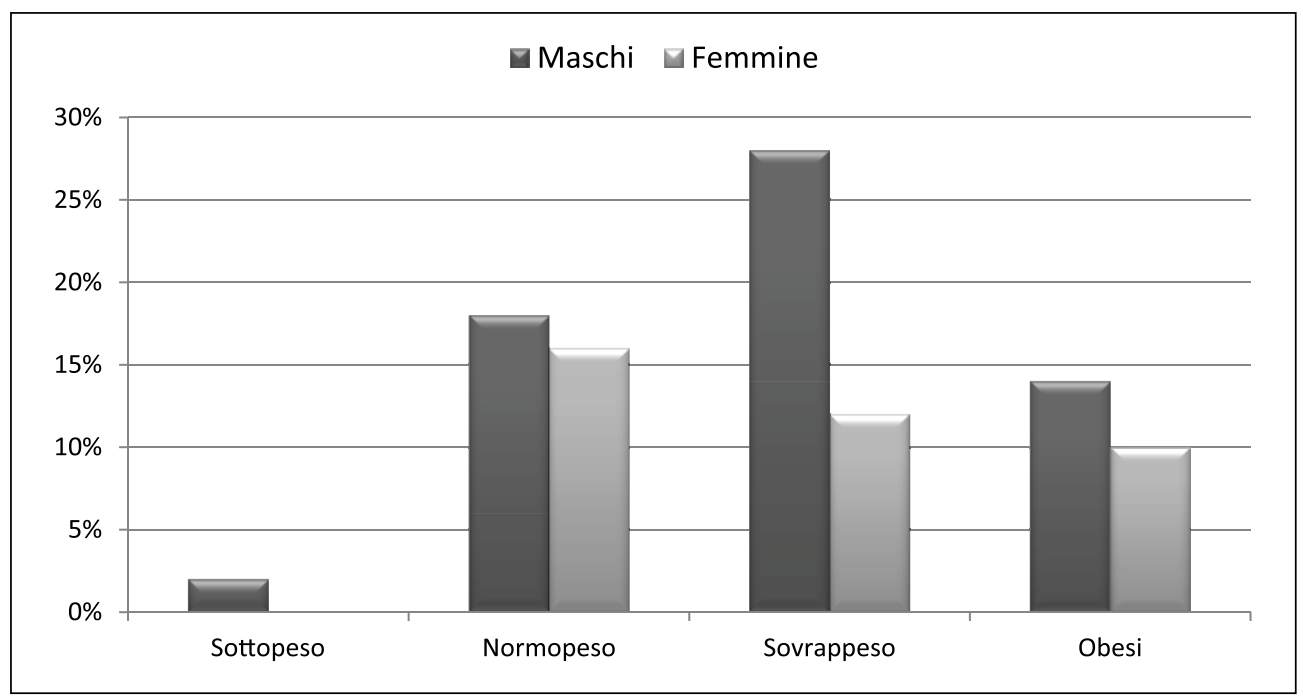

Figura I. Distribuzione dell'Indice di Massa Corporea nei pazienti portatori di trapianto renale divisi per genere.

Tabella 2. Introiti nutrizionali nei pazienti portatori di trapianto renale; popolazione totale, divisa per genere e in base ai terzili di funzione renale.

\begin{tabular}{|c|c|c|c|c|c|c|c|c|c|}
\hline & & Totale & Maschi & Femmine & $P$ & eGFR $>66.7$ & eGFR 44.6-66.7 & eGFR $<44.6$ & $\mathrm{P}$ \\
\hline Energia & kcal & $1967 \pm 433$ & $2150 \pm 349$ & $1668 \pm 394$ & 0.000 & $|78| \pm 353$ & $2070 \pm 446$ & $2040 \pm 455$ & 0.113 \\
\hline Energia & kcal/kg & $27.6 \pm 7.6$ & $28.4 \pm 6.6$ & $26.3 \pm 8.9$ & 0.348 & $26.2 \pm 7.4$ & $28.0 \pm 8$ & $28.4 \pm 7.5$ & 0.669 \\
\hline Proteine & $\%$ & $15.7 \pm 2.9$ & $15.4 \pm 2.2$ & $16.3 \pm 3.8$ & 0.261 & $16.9 \pm 4.4$ & $15.2 \pm 1.9$ & $15.2 \pm 1.9$ & 0.155 \\
\hline Grassi & $\%$ & $32.6 \pm 5.9$ & $32.9 \pm 6.2$ & $32.2 \pm 5.7$ & 0.690 & $32 \pm 5$ & $32.6 \pm 7.9$ & $33.3 \pm 5$ & 0.837 \\
\hline Carboidrati & $\%$ & $51.7 \pm 6.3$ & $51.8 \pm 6.5$ & $51.5 \pm 6.2$ & 0.895 & $51.2 \pm 6.9$ & $52.2 \pm 7$ & $51.6 \pm 6.3$ & 0.901 \\
\hline Proteine & g/die & $75.6 \pm 16.2$ & $80.6 \pm 16.2$ & $67.3 \pm 21.1$ & 0.015 & $73.9 \pm 21.6$ & $77.8 \pm 18.6$ & $74.8 \pm 18.3$ & 0.846 \\
\hline Proteine & $\mathrm{g} / \mathrm{kg} / \mathrm{die}$ & $1.06 \pm 0.32$ & $1.06 \pm 0.28$ & $1.04 \pm 0.38$ & $0.85 I$ & $1.06 \pm 0.35$ & $1.06 \pm 0.35$ & $1.04 \pm 0.28$ & 0.974 \\
\hline Grassi & g/die & $70.4 \pm 19.1$ & $76.2 \pm 15.4$ & $60.9 \pm 21.2$ & 0.005 & $63.6 \pm 16.9$ & $74.1 \pm 21.5$ & $70.4 \pm 19.1$ & 0.232 \\
\hline Saturi & g/die & $15.5 \pm 6.0$ & $16.7 \pm 5.3$ & $13.6 \pm 6.7$ & 0.073 & $13.4 \pm 5.3$ & $17.2 \pm 5.7$ & $15.9 \pm 6.0$ & 0.184 \\
\hline Monoinsaturi & g/die & $32.8 \pm 9.9$ & $35.5 \pm 7.9$ & $28.3 \pm 11.4$ & 0.012 & $29.5 \pm 10$ & $34.6 \pm 10.7$ & $34.0 \pm 8.6$ & 0.274 \\
\hline Polinsaturi & $\mathrm{g} / \mathrm{die}$ & $6.5 \pm 2.3$ & $7.0 \pm 2.1$ & $5.6 \pm 2.4$ & 0.025 & $5.8 \pm 2.5$ & $7.2 \pm 2.6$ & $6.5 \pm 1.7$ & 0.243 \\
\hline Colesterolo & $\mathrm{mg} / \mathrm{die}$ & $193 \pm 81$ & $213 \pm 86$ & $159 \pm 84$ & 0.035 & $153 \pm 69$ & $231 \pm 106 *$ & $194 \pm 75$ & 0.043 \\
\hline Carboidrati & g/die & $267 \pm 69$ & $292 \pm 68$ & $225 \pm 48$ & 0.000 & $24 I \pm 58$ & $285 \pm 75$ & $274 \pm 70$ & 0.182 \\
\hline Zuccheri & $\mathrm{g} / \mathrm{die}$ & $67.8 \pm 30.7$ & $67.1 \pm 24.0$ & $69.1 \pm 39.9$ & 0.828 & $71.9 \pm 42.3$ & $68.2 \pm 28.5$ & $63.9 \pm 19.6$ & 0.757 \\
\hline Fibre & g/die & $12.2 \pm 4.9$ & $11.9 \pm 5.5$ & $12.6 \pm 4.0$ & 0.908 & $12.1 \pm 4.5$ & $12.6 \pm 4.8$ & $11.9 \pm 6.6$ & 0.932 \\
\hline Calcio & $\mathrm{mg} / \mathrm{die}$ & $484 \pm 236$ & $504 \pm 257$ & $450 \pm 199$ & 0.660 & $510 \pm 210$ & $498 \pm 281$ & $447 \pm 223$ & 0.719 \\
\hline Sodio & $\mathrm{mg} / \mathrm{die}$ & $1203 \pm 679$ & $|25| \pm 746$ & $1125 \pm 563$ & 0.529 & $1052 \pm 580$ & $1267 \pm 753$ & $1282 \pm 679$ & 0.565 \\
\hline Potassio & $\mathrm{mg} / \mathrm{die}$ & $1929 \pm 487$ & $2032 \pm 474$ & $1760 \pm 470$ & 0.054 & $1914 \pm 512$ & $1955 \pm 474$ & $1918 \pm 501$ & 0.967 \\
\hline Fosforo & $\mathrm{mg} / \mathrm{die}$ & $1047 \pm 364$ & $106 \mid \pm 203$ & $1025 \pm 539$ & 0.739 & $995 \pm 210$ & $1030 \pm 265$ & $1109 \pm 523$ & 0.653 \\
\hline Ferro & $\mathrm{mg} / \mathrm{die}$ & $9.6 \pm 2.9$ & $10.5 \pm 2.8$ & $8.2 \pm 2.4$ & 0.004 & $8.5 \pm 2.3$ & $10.2 \pm 3.4$ & $9.6 \pm 2.9$ & 0.185 \\
\hline
\end{tabular}

dunque con elevato rischio cardiovascolare. ${ }^{1,2}$ Tra i vari fattori che possono aumentare il rischio di sovrappeso/ obesità nel TxR vi è l'uso di steroidi; per valutare questa possibile relazione abbiamo correlato la durata del trapianto (misura indiretta ma altamente predittiva della dose cumulativa di steroide) e l'IMC: la correlazione non ha dato risultati significativi. Dunque, la dose di steroidi non sembra avere una relazione con l'IMC suggerendo che altri fattori possano avere un ruolo importante sull'aumento dell'IMC nel trapianto renale.
Le linee guida raccomandano un introito energetico compreso tra $25-30 \mathrm{kcal} / \mathrm{kg}$ di peso dopo le prime 5 settimane dal trapianto, così come per la popolazione generale. L'apporto proteico raccomandato è invece di $1 \mathrm{~g} / \mathrm{kg}$ di peso corporeo. I nostri risultati suggeriscono una tendenza ad un apporto calorico nella media, senza significative differenze di genere o per livello di funzionalità renale, con un introito proteico per grado di funzione renale elevato nella MRC di grado moderato e avanzato e con una distribuzione dei macronutrienti piuttosto equilibrata; risultati simili sono 


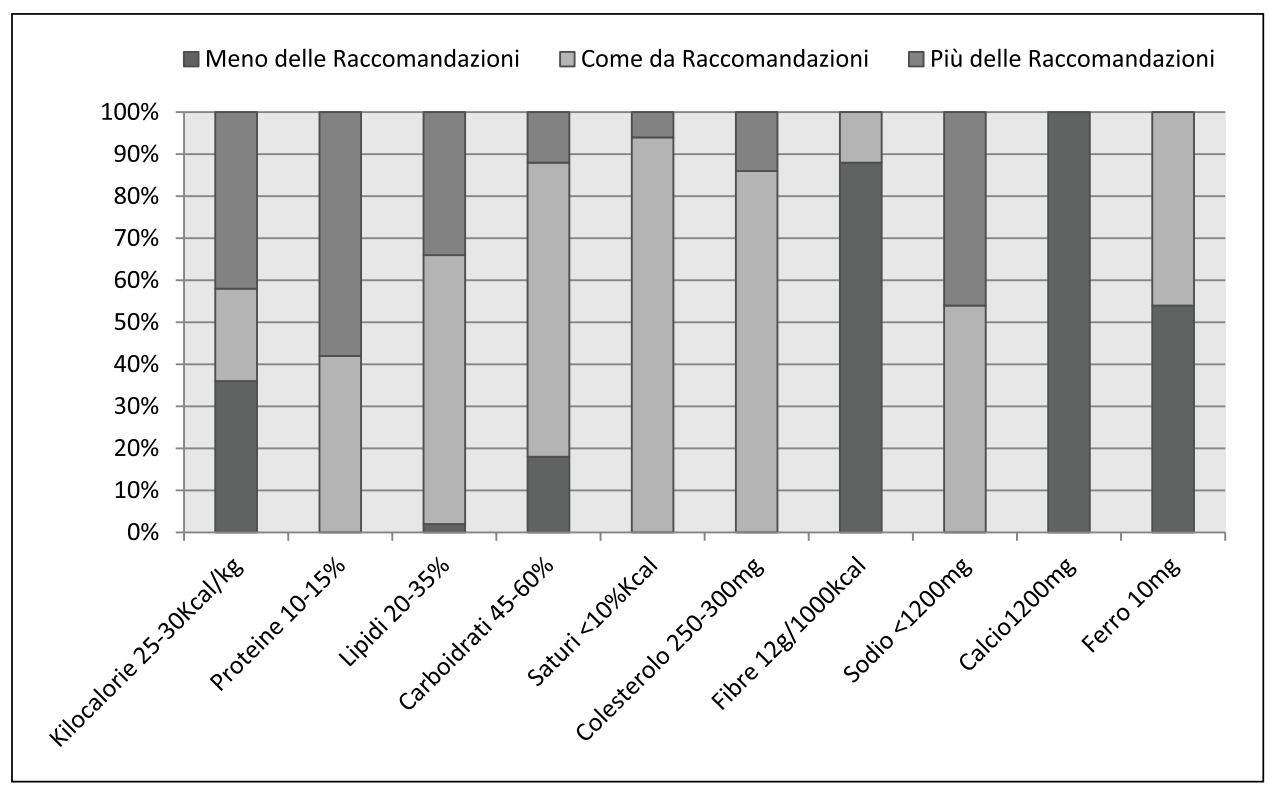

Figura 2. Distribuzione degli introiti di nutrienti nei pazienti portatori di trapianto renale in relazione ai livelli raccomandati nella popolazione generale.

Tabella 3. Terapia immunosoppressiva nel totale dei pazienti portatori di trapianto renale e in base ai terzili di funzione renale.

\begin{tabular}{lcccc}
\hline & Totale & eGFR $>66.7$ & eGFR 44.6-66.7 & eGFR $<44.6$ \\
\hline Numero Farmaci, \% $p z$ & & & & 0 \\
I & 0 & 0 & 4 & 0 \\
2 & 8 & 4 & 24 & 36 \\
3 & 88 & 28 & 0 & 2 \\
4 & 4 & 2 & & 36 \\
Classe Farmaci, \% pz & 100 & 34 & 16 & 28 \\
Steroidi & 52 & 10 & 10 & 12 \\
Ciclosporina & 44 & 22 & 22 & 36 \\
Tacrolimus & 80 & 22 & 2 & 2 \\
MMF/MPA & 10 & 6 & & \\
Everolimus & & & & \\
\hline
\end{tabular}

stati riportati anche in un' altra pubblicazione. ${ }^{21}$ Focalizzando l'attenzione sull'apporto di micronutrienti e fibre, si conferma quanto descritto in precedenza ${ }^{22,23}$ e cioè che l'apporto di sodio supera largamente i livelli raccomandati, mentre la quasi totalità del campione non soddisfa i fabbisogni di calcio, fibre e ferro, specie nelle femmine. Allo scopo di valutare se l'introito di ferro possa avere una relazione con il grado di anemia, abbiamo fatto una correlazione tra introito di ferro e livelli di emoglobina in tutti i pazienti, ma nessuna correlazione significativa è stata rilevata e, dunque, altri fattori sembrano avere un ruolo maggiore sull'anemia nel paziente con TxR.

In conclusione, $\mathrm{i}$ risultati di questo studio indicano diversi difetti alimentari, in eccesso o anche in difetto, che possono avere ripercussioni sui principali fattori di rischio nei pazienti con TxR. Questi dati devono essere confermati in popolazioni più ampie di TxR, analizzando anche le influenze del contesto sociale e dei determinanti dei diversi comportamenti alimentari nella popolazione dei TxR. In ogni caso, una maggiore attenzione deve essere posta sull'assunzione delle proteine e di alcuni micronutrienti tra cui sodio, calcio e ferro. E' pertanto consigliabile che $i$ pazienti con TxR vengano sottoposti regolarmente a un counselling nutrizionale da parte di un dietista renale e che vengano studiati periodicamente per valutare lo stato di nutrizione e gli apporti alimentari.

\section{Dichiarazione di assenza di conflitto di interessi}

Gli Autori dichiarano di non avere conflitti di interessi.

\section{Finanziamenti}

Gli Autori dichiarano di non aver ricevuto finanziamenti specifici da qualsiasi ente nei settori pubblico, privato o senza fini di lucro. 


\section{Bibliografia}

1. Ojo, A. O. Cardiovascular complications after renal transplantation and their prevention. Transplantation 2006; 82: 603-611.

2. Aakhus, S., Dahl, K. Widerøe, T. E. Cardiovascular disease in stable renal transplant patients in Norway: Morbidity and mortality during a 5-yr follow-up. Clin Transplant 2004; 18: 596-604.

3. Aakhus, S., Dahl, K. \& Wideroe, T. E. Cardiovascular morbidity and risk factors in renal transplant patients. Nephrol Dial Transpl 1999; 14: 648-654.

4. Teplan, V. et al. Nutritional Consequences of Renal Transplantation. J Ren Nutr 2009; 19: 95-100.

5. Guida, B., Cataldi, M., Maresca, ID. et al. Dietary intake as a link between obesity, systemic inflammation, and the assumption of multiple cardiovascular and antidiabetic drugs in renal transplant recipients. Biomed Res Int 2013. DOI: $10.1155 / 2013 / 363728$

6. Kasiske, B. et al. K/DOQI clinical practice guidelines for managing dyslipidemias in chronic kidney disease. Am. J. Kidney Dis 2003; 41: S91.

7. Lopes, I. M., Martín, M., Errasti, P. et al. Benefits of a dietary intervention on weight loss, body composition, and lipid profile after renal transplantation. Nutrition 1999; 15: 7-10.

8. Zha, Y. Qian, Q. Protein nutrition and malnutrition in CKD and ESRD. Nutrients 2017; 27: 9.

9. Battaglia, Y., Galeano, D., Cojocaru, E. et al. Muscle-wasting in end stage renal disease in dialysis treatment: a review. G. Ital. Nefrol 2016; 33(2).

10. Chan, M. et al. Evidence-based guidelines for the nutritional management of adult kidney transplant recipients. Journal of Renal Nutrition 2011; 21: 47-51.

11. Beto, J. A., Ramirez, W. E. \& Bansal, V. K. Medical nutrition therapy in adults with chronic kidney disease: Integrating evidence and consensus into practice for the generalist registered dietitian nutritionist. J Acad Nutr Diet 2014; 114: 1077-1087.

12. Martins, C., Pecoits-Filho, R. Riella, M. C. Nutrition for the post-renal transplant recipients. in Transplantation Proceedings 2004; 36: 1650-1654.

13. Bellizzi, V., Cupisti, A., Locatelli, F. et al. Low-protein diets for chronic kidney disease patients: the Italian experience. BMC Nephrol 2016; 17(1): 77.

14. Cupisti, A. et al. Nutrition and physical activity in CKD patients. Kidney and Blood Pressure Research 2014; 39: 107-113.

15. D'Alessandro, C., Piccoli, G., Calella, P. et al. 'Dietaly': practical issues for the nutritional management of CKD patients in Italy. BMC Nephrol 2016; 17(1): 102.

16. Tabelle LARN 2014, http://www.sinu.it/html/pag/tabelle larn_2014_rev.asp 2012; 22-23 (consultato: 27 novembre 2018).

17. WHO. WHO: Global Database on Body Mass Index. World Health Organisation. 2015.

18. Johnson, D. W. et al. The effect of obesity on renal transplant outcomes. Transplantation 2002; 74: 675-681.

19. Rettkowski, O., Wienke, A., Hamza, A. et al. Low Body Mass Index in Kidney Transplant Recipients: Risk or Advantage for Long-Term Graft Function? Transplant. Proc. 2007; 39: 1416-1420.

20. Djukanović, L. et al. Co-morbidity and kidney graft failuretwo main causes of malnutrition in kidney transplant patients. Nephrol. Dial. Transplant 2003; 18: 68-72.

21. Sasaki, H. et al. Nutritional status in Japanese renal transplant recipients with long-term graft survival. Transplant. Proc. 2015; 47: 367-372.

22. Kalbfleisch, J. H., Hebert, L. A., Lemann, J. et al. Habitual excessive dietary salt intake and blood pressure levels in renal transplant recipients. Am. J. Med. 1982; 73: 205-210.

23. Moeller, T., Buhl, M., Schorr, U. et al. Salt intake and hypertension in renal transplant patients. Clin. Nephrol. 2000; 53: 159-163. 